\title{
The Closure Condition of the Double Banana and Its Application to Robot Position Analysis
}

\author{
Nicolás Rojas and Federico Thomas
}

\begin{abstract}
A double banana is defined as the bar-and-joint assembly of two bipyramids joined by their apexes. Clearly, the bar lengths of this kind of assembly are not independent as we cannot assign arbitrary values to them. This dependency can be algebraically expressed as a closure condition fully expressed in terms of bar lengths. This paper is devoted to its derivation and to show how its use simplifies the position analysis of many wellknown serial and parallel robots thus providing a unifying treatment to apparently disparate problems. This approach permits deriving the univariate polynomials, needed for the closed-form solution of these position analysis problems, without relying on trigonometric substitutions or difficult variable eliminations.
\end{abstract}

$$
\begin{aligned}
& P_{i} \quad \text { A point in } \mathbb{E}^{3} \\
& \mathbf{p}_{i} \quad \text { Position vector of point } P_{i} \text { with respect to a }
\end{aligned}
$$

\section{INTRODUCTION}

The bar-and-joint assembly resulting from linking two bipyramids by their apical vertices is commonly known as a double banana (see Fig. 11). Since the two bipyramids are free to rotate about the line joining the common apexes, the double banana has become the classic example of a bar-andjoint assembly that satisfies Maxwell's rule for the rigidity of frames and yet is clearly flexible [1].

The solution of the inverse kinematics of serial-chain robots and the direct kinematics of in-parallel platforms are usually considered within the area of position analysis kinematics (or kinematic geometry) [2]. These problems, when solved in closed-form, require finding univariate polynomials whose roots determine the sought solutions (commonly known as assembly modes). These easy-to-formulate but, in general, difficult-to-solve problems have received the attention of many roboticians but the techniques developed to solve them mainly rely on the computation of kinematically

This work was supported by the Spanish Ministry of Economy and Competitiveness through the Explora programme under Contract DPI201113208-E.

The authors are with the Institut de Robòtica i Informàtica Industrial (CSIC-UPC), Llorens Artigas, 4-6, 08028, Barcelona, Spain \{nrojas, fthomas\}airi.upc.edu

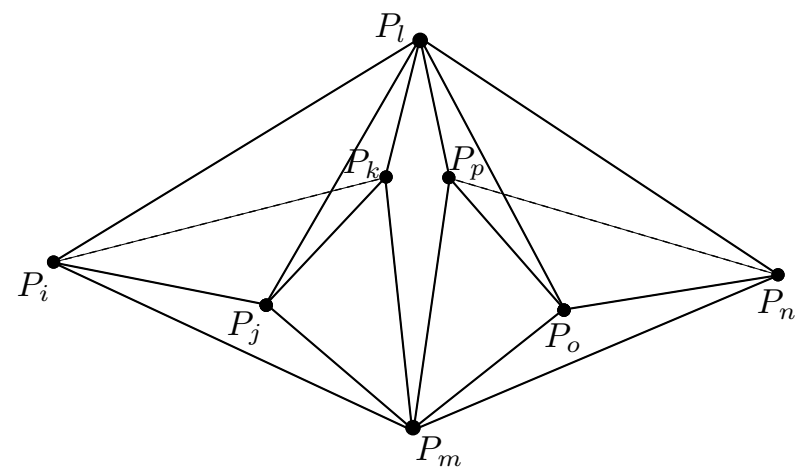

Fig. 1. A double banana is a bar-and-joint assembly defined by two bipyramids joined by their apexes $\left(P_{l}\right.$ and $\left.P_{m}\right)$. This assembly is not rigid because the two bipyramids can rotate about the axis defined by the common apexes.

independent loop equations that are solved using the same techniques to solve arbitrary sets of non-linear equations [3]. The aim of this paper is to show that alternative formulations to those based on loop equations are possible, and that their use can provide significant advantages including the unnecessity, in many non-trivial cases, of variable eliminations to derive the final univariate polynomials.

This paper is organized as follows. Section II presents some basic facts on Cayley-Menger determinants that are used in Section III to derive the closure condition of the double banana. Section IV applies this closure condition to solve the position analysis of well-known non-trivial robots such as the inverse kinematics of the $3 \mathrm{R}$ serial robot or the forward kinematics of the octahedral manipulator. Section $\mathrm{V}$ presents a numerical example for the forward kinematics of decoupled Stewart platform. Finally, we conclude in Section VI.

\section{CAyley-Menger Determinants}

The Cayley-Menger bi-determinant of the point sequences $P_{i_{1}}, \ldots, P_{i_{n}}$, and $P_{j_{1}}, \ldots, P_{j_{n}}$ is defined as:

$D\left(i_{1}, \ldots, i_{n} ; j_{1}, \ldots, j_{n}\right)=2\left(\frac{-1}{2}\right)^{n}\left|\begin{array}{cccc}0 & 1 & \ldots & 1 \\ 1 & s_{i_{1}, j_{1}} & \ldots & s_{i_{1}, j_{n}} \\ \vdots & \vdots & \ddots & \vdots \\ 1 & s_{i_{n}, j_{1}} & \ldots & s_{i_{n}, j_{n}}\end{array}\right|$.

When the two point sequences are the same, it is convenient to abbreviate $D\left(i_{1}, \ldots, i_{n} ; i_{1}, \ldots, i_{n}\right)$ by $D\left(i_{1}, \ldots, i_{n}\right)$, which is simply called the Cayley-Menger determinant of the involved point sequence. 
The evaluation of $D\left(i_{1}, \ldots, i_{n}\right)$ gives $(n-1) !^{2}$ times the squared hypervolume of the simplex spanned by the points $P_{i_{1}}, \ldots, P_{i_{n}}$ in $\mathbb{E}^{n-1}$ [4, pp. 737-738]. Then, we can introduce the following two definitions:

- $A_{i, j, k}=+\frac{1}{2} \sqrt{D(i, j, k)}$ to denote the area of the triangle defined by $P_{i}, P_{j}$, and $P_{k}$.

- $V_{i, j, k, l}= \pm \frac{1}{6} \sqrt{D(i, j, k, l)}$ to denote the oriented volume of the tetrahedron defined by $P_{i}, P_{j}, P_{k}$, and $P_{l}$. It is defined as positive if $\left|\mathbf{p}_{i, j}, \mathbf{p}_{i, k}, \mathbf{p}_{i, l}\right|>0$, and negative otherwise.

Observe that, when working in $\mathbb{E}^{n}$, all Cayley-Menger determinants involving more than $n+2$ points necessarily vanish. For more properties of Cayley-Menger determinants, the interested reader is referred to [5], [6], [7].

Many geometric problems can be elegantly formulated using Cayley-Menger determinants — see, for instance, [5] and the examples therein. Next, we show how the computation of the double banana closure condition is one of them.

\section{THE DOUBLE BANANA CLOSURE CONDITION}

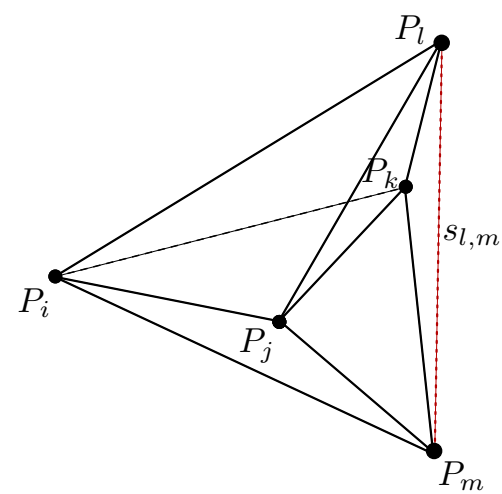

Fig. 2. The squared distance between the apexes of a bipyramid, in this case $s_{l, m}$, can be expressed in terms of the bipyramid squared edge lengths using Cayley-Menger determinants.

A bipyramid is a set of two tetrahedra sharing one face (Fig. 2). The bar-and-joint framework defined by the edges of a bipyramid is rigid because the joints cannot be moved continuously while preserving the bar lengths.

The joint centers of this framework defines a simplex in $\mathbb{E}^{4}$. Then, since it is actually embedded in $\mathbb{E}^{3}$, its volume is null. Therefore, according to the notation of Fig. 2, we have that

$$
D(i, j, k, l, m)=0 .
$$

The expansion of the above equation yields a quadratic expression in $s_{l, m}$, the square distance between the apexes of the bipyramid. By solving it, we obtain the two feasible values for $s_{l, m}$.

By applying Jacobi's theorem to a partition of the determinant $D(i, j, k, l, m)$ (see [8] for details), it can be proved that:

$$
\begin{aligned}
& D(i, j, k, l, m)= \\
& \quad \frac{D(i, j, k, l) D(i, j, k, m)-D^{2}(i, j, k, l ; i, j, k, m)}{D(i, j, k)}=0 .
\end{aligned}
$$

Then, assuming that $P_{i}, P_{j}$, and $P_{k}$ are not aligned (i.e., $D(i, j, k) \neq 0)$,

$$
D(i, j, k, l ; i, j, k, m)= \pm \sqrt{D(i, j, k, l) D(i, j, k, m)} .
$$

Since the left hand side of the above equation is linear in $s_{l, m}$, it can be expressed as

$$
\begin{gathered}
D(i, j, k, l ; i, j, k, m)=\frac{\partial D(i, j, k, l ; i, j, k, m)}{\partial s_{l, m}} s_{l, m} \\
+\left.D(i, j, k, l ; i, j, k, m)\right|_{s_{l, m}=0} \\
=-\frac{1}{2} D(i, j, k) s_{l, m}+\left.D(i, j, k, l ; i, j, k, m)\right|_{s_{l, m}=0}
\end{gathered}
$$

Therefore, substituting (4) in (3), we conclude that

$$
\begin{aligned}
s_{l, m}=\frac{2}{D(i, j, k)}( & \left.D(i, j, k, l ; i, j, k, m)\right|_{s_{l, m}=0} \\
& \pm \sqrt{D(i, j, k, l) D(i, j, k, m)}),
\end{aligned}
$$

which can be rewritten as:

$$
s_{l, m}=\frac{1}{2 A_{i, j, k}^{2}}\left(\Psi_{i, j, k, l, m}+36 V_{i, j, k, l} V_{i, j, k, m}\right),
$$

where $\Psi_{i, j, k, l, m}=\left.D(i, j, k, l ; i, j, k, m)\right|_{s_{l, m}=0}$.

Finally, by considering separately the two constituent bipyramids of the double banana in Fig. 1, we have that

$$
\begin{aligned}
& s_{l, m}=\frac{1}{2 A_{i, j, k}^{2}}\left(\Psi_{i, j, k, l, m}+36 V_{i, j, k, l} V_{i, j, k, m}\right), \\
& s_{l, m}=\frac{1}{2 A_{n, o, p}^{2}}\left(\Psi_{n, o, p, l, m}+36 V_{n, o, p, l} V_{n, o, p, m}\right) .
\end{aligned}
$$

Therefore, by equating the right hand side of the above two equations, we conclude that:

$$
\begin{gathered}
A_{n, o, p}^{2}\left(\Psi_{i, j, k, l, m}+36 V_{i, j, k, l} V_{i, j, k, m}\right) \\
-A_{i, j, k}^{2}\left(\Psi_{n, o, p, l, m}+36 V_{n, o, p, l} V_{n, o, p, m}\right)=0
\end{gathered}
$$

This equation is the closure condition of a double banana. It is satisfied if, and only if, it can be assembled with the assigned bar lengths.

Next, it is shown how, by using this closure condition, univariate polynomials for the position analysis of important serial and parallel robots can be straightforwardly obtained without relying on trigonometric substitutions or difficult variable eliminations 


\section{Applications to Robot Position Analysis}

\section{$A$. Inverse kinematics of $3 R$ serial robots}

Fig. 3 top) shows a general 3R serial robot, a manipulator with three rotational joint variables. This robot has been used for positioning tasks in the Cartesian space or as the regional serial kinematic chain in wrist-partitioned $6 \mathrm{R}$ robots, the most common serial robots [9], [10].
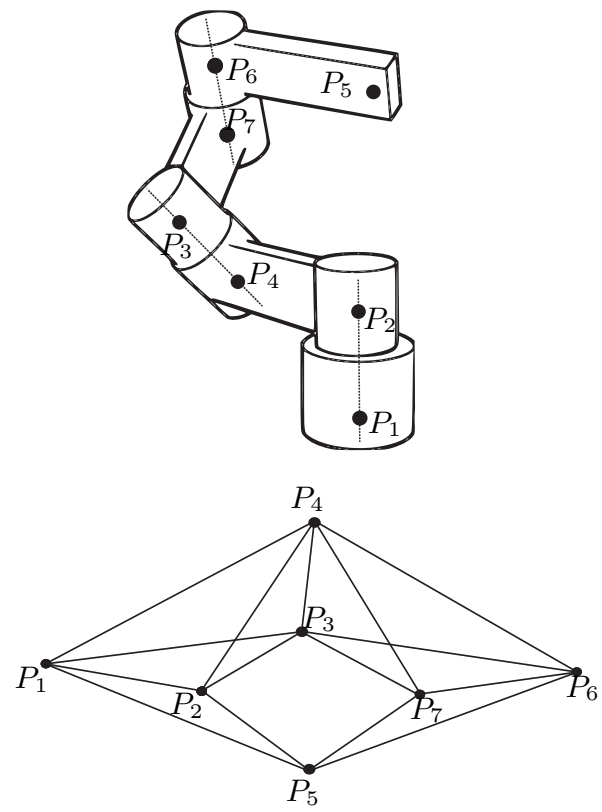

Fig. 3. A 3R robot (top), and its kinematically equivalent bar-and-joint assembly (bottom).

The inverse kinematics problem of this serial robot consists in finding the values of its joint angles to attain a given location for its end-effector relative to the base. It has been shown that this problem reduces to compute the solutions of a fourth-order polynomial. This quartic was obtained for the first time by Pieper and Roth in [11] using homogeneous transformations. In [12], Smith and Lipkin analyzed geometrically this solution using conic sections. Next we present an alternative to the approach based on homogeneous transformations that exploits the closure condition of the double banana derived in the previous section.

In a serial manipulator, a link connecting two skew revolute axes can be modelled by taking two points on each of these axes and connecting them all with edges to form a tetrahedron [13]. Then, a 3R serial robot can be modelled as the bar-and-joint framework involving 7 joints and 15 bars shown in Fig. 3(bottom). Observe how such bar-and-joint framework corresponds to the double banana in Fig. 1 after removing the bars linking $P_{p}$ and $P_{m}$, and $P_{k}$ and $P_{m}$, and then merging $P_{k}$ and $P_{p}$. Therefore, according to the notation of Fig. 3 and equation (6), the closure condition of the 3R serial robot is given by

$$
\begin{aligned}
& A_{6,7,3}^{2}\left(\Psi_{1,2,3,4,5}+36 V_{1,2,3,4} V_{1,2,3,5}\right) \\
-A_{1,2,3}^{2}\left(\Psi_{6,7,3,4,5}+36 V_{6,7,3,4} V_{6,7,3,5}\right) & =0 .
\end{aligned}
$$

This equation is a scalar radical equation in a single variable: $s_{3,5}$. The different real roots of this equation - values of $s_{3,5}$ - correspond to the different solutions of the inverse kinematics problem. The above equation can be reduced to a polynomial by simply clearing the radicals associated with $V_{1,2,3,5}$ and $V_{6,7,3,5}$. This yields

$$
\begin{aligned}
& \left(A_{6,7,3}^{4} \Psi_{1,2,3,4,5}^{2}-2 A_{6,7,3}^{2} A_{1,2,3}^{2} \Psi_{1,2,3,4,5} \Psi_{6,7,3,4,5}\right. \\
& +A_{1,2,3}^{4} \Psi_{6,7,3,4,5}^{2}+1296 A_{6,7,3}^{4} V_{1,2,3,4}^{2} V_{1,2,3,5}^{2} \\
& \left.-1296 A_{1,2,3}^{4} V_{6,7,3,4}^{2} V_{6,7,3,5}^{2}\right)^{2}-\left(72 A_{6,7,3}^{4} \Psi_{1,2,3,4,5}\right. \\
& \left.V_{1,2,3,4}-72 A_{1,2,3}^{2} A_{6,7,3}^{2} V_{1,2,3,4} \Psi_{6,7,3,4,5}\right)^{2} V_{1,2,3,5}^{2}=0 .
\end{aligned}
$$

The expansion of the above equation leads to a fourthdegree polynomial in $s_{3,5}$, the univariate polynomial for the inverse kinematics of the $3 \mathrm{R}$ serial robot. Observe how this polynomial is obtained without relying on variable eliminations or trigonometric substitutions. For each of the real roots of equation (8), vectors $\mathbf{p}_{3,4}$ and $\mathbf{p}_{6,7}$ can be determined by a sequence of trilaterations as follows (see [7] for a vector formulation of trilateration):

- $\mathbf{p}_{1,3}$ can be obtained from $\mathbf{p}_{1,2}$ and $\mathbf{p}_{1,5}$, then

- $\mathbf{p}_{1,4}$ from $\mathbf{p}_{1,2}$ and $\mathbf{p}_{1,3}$, then

- $\mathbf{p}_{4,7}$ from $\mathbf{p}_{4,3}$ and $\mathbf{p}_{4,5}$, and finally

- $\mathbf{p}_{4,6}$ from $\mathbf{p}_{4,3}$ and $\mathbf{p}_{4,7}$.

This procedure leads to up to four locations for $P_{6}$ and at least one of them must satisfy the distance imposed between $P_{5}$ and $P_{6}$.

\section{B. The forward kinematics of a 4-3 Stewart platform}

Consider the Stewart platform with topology \& \& (see Fig. 4). It is known that this parallel robot can adopt up to 8 assembly configurations [14].

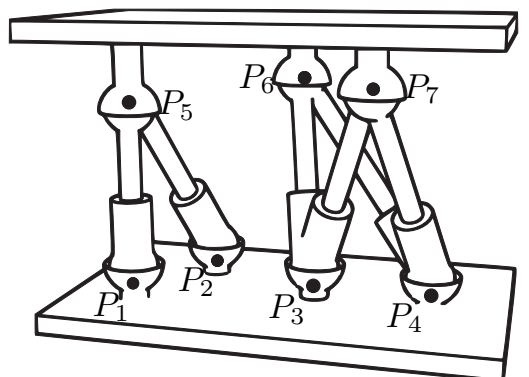

Fig. 4. 4-3 Stewart platform whose kinematically equivalent bar-and-joint assembly is the same as that of a $3 R$ robot.

Observe how the bar-and-joint framework associated with this parallel robot is exactly the same as that associated with the 3R serial robot analyzed in the previous subsection. Therefore, equation (8) also corresponds to the univariate polynomial for the forward kinematics of this 4-3 Stewart platform.

For each of the real roots of equation (8), the feasible locations of the three points defining the moving platform can be determined by a sequence of trilaterations as follows:

- $\mathbf{p}_{3,5}$ can be obtained from $\mathbf{p}_{3,2}$ and $\mathbf{p}_{3,1}$, then 
- $\mathbf{p}_{3,7}$ from $\mathbf{p}_{3,5}$ and $\mathbf{p}_{4,5}$, and finally

- $\mathbf{p}_{3,6}$ from $\mathbf{p}_{3,7}$ and $\mathbf{p}_{3,5}$.

This procedure leads to up to eight locations for $P_{6}$.
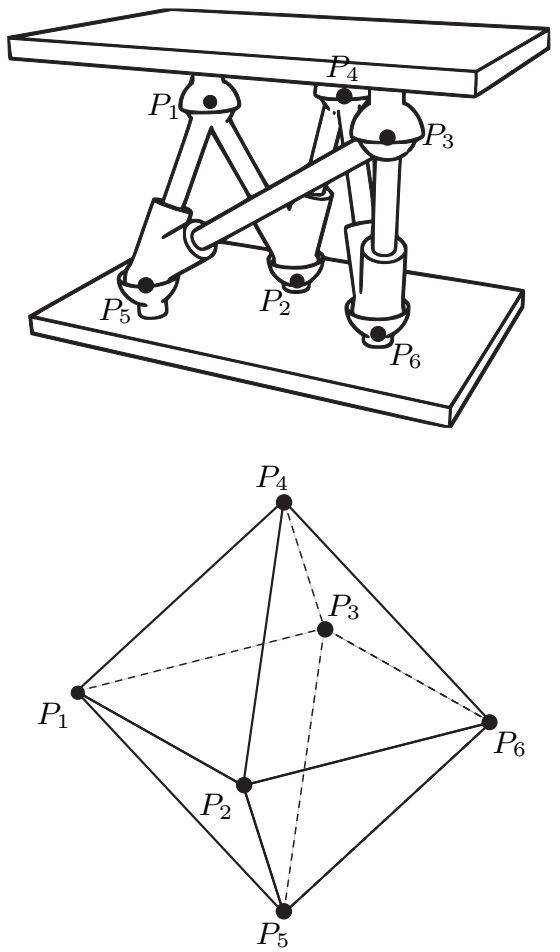

Fig. 5. The octahedral Stewart platform (top) and its kinematically equivalent bar-and-joint assembly (bottom).

\section{Forward kinematics of the octahedral Stewart platform}

Figure 5(top) shows an octahedral Stewart platform. During the late 80's and early 90's several researchers successfully addressed the forward kinematics problem of this platform in closed form, giving procedures that reduce the problem to finding the roots of an eighth-degree univariate polynomial. In [15], Nanua et al. derived such polynomial through resultant elimination and tangent-half-angle substitution techniques. A similar result, based on three spherical four-bar linkages, was obtained by Griffis and Duffy in [16]. An alternative method was also developed by Innocenti and Parenti-Castelli in [17]. Michelucci and Foufou, using Cayley-Menger determinants, obtained an algebraic system of two equation in two unknowns from which, using Sylvester elimination, a resultant of degree 16 in one unknown was derived [6]. More recently, Akçali and Mutlu revisited the problem - also using resultant elimination and tangent-half-angle substitution techniques - with the aim of reducing the computational cost of evaluating the resulting univariate polynomial [18].

Alternatively to the above approaches, using the closure condition for the double banana, a simple procedure for solving the forward kinematics problem of the octahedral manipulator is obtained. Indeed, the kinematically equivalent bar-and-joint framework to this platform appears in Fig. 5 (bottom). It corresponds to a double banana in which, as labelled in Fig. 1, bars joining $P_{j}$ and $P_{k}$, and $P_{p}$ and $P_{o}$, have been removed and then joints $P_{k}$ and $P_{p}$, and $P_{j}$ and $P_{o}$, have been merged. Therefore, according to the notation of Fig. 5 top) and equation (6), the closure condition of the octahedral platform is

$$
\begin{aligned}
& A_{6,2,3}^{2}\left(\Psi_{1,2,3,4,5}+36 V_{1,2,3,4} V_{1,2,3,5}\right) \\
& -A_{1,2,3}^{2}\left(\Psi_{6,2,3,4,5}+36 V_{6,2,3,4} V_{6,2,3,5}\right)=0 .
\end{aligned}
$$

This equation is a scalar radical equation in a single variable: $s_{2,3}$. It is satisfied if, and only if, the considered octahedral manipulator can be assembled. Actually, its different real roots correspond to the different solutions of the forward kinematics problem.

Similarly to the case of the $3 \mathrm{R}$ serial robot, equation (9) can be reduced to a polynomial by clearing the radicals associated with $V_{1,2,3,4}, V_{1,2,3,5}, V_{6,2,3,4}$, and $V_{6,2,3,5}$. This procedure yields an eighth-degree univariate polynomial in $s_{2,3}$. Observe again how this polynomial is obtained without relying on variable eliminations or trigonometric substitutions. For each of the real roots of this polynomial, the feasible locations of the three points defining the moving platform can be determined by a sequence of trilaterations as follows:

- $\mathbf{p}_{2,3}$ from $\mathbf{p}_{2,5}$ and $\mathbf{p}_{2,6}$, then

- $\mathbf{p}_{2,4}$ from $\mathbf{p}_{2,3}$ and $\mathbf{p}_{2,6}$, and finally

- $\mathbf{p}_{2,1}$ from $\mathbf{p}_{2,3}$ and $\mathbf{p}_{2,4}$.

This procedure leads to up to eight locations for $P_{1}$ and at least one of them must satisfy the distance imposed between $P_{1}$ and $P_{5}$.

The study of the octahedral manipulator degeneracies [19] is not treated here but their analysis could probably benefit from the simplicity of the closure condition (9).

\section{Forward kinematics of the decoupled Stewart platform}

Consider the Stewart platform with topology ise appearing in Fig. 6(top). This robot is a specialization of the general decoupled Stewart platform whose topology is $\therefore$ This robot is also known as the spherical Stewart platform [20, p. 114], a robot with position and orientation driven by independent active prismatic joints and one of the typical topologies used to obtain parallel wrists [21]. It has been proved that the parallel robots with these topologies can adopt up to 16 assembly configurations [14]. A closed-form solution for the forward kinematics of the considered robot was obtained for the first time by Innocenti and ParentiCastelli by using resultant elimination and tangent-halfangle substitution techniques [22]. Later on, other univariate polynomials were presented at least by Wohlhart [23] and Husain and Waldron [24].

The kinematically equivalent bar-and-joint framework to the Stewart platform in Fig. 6 (top) appears in Fig. 6(bottom). The following three double bananas can be identified in this framework:

1) The one resulting from removing joint $P_{1}$ : a double banana in which, as labelled in Fig. 1, bars linking 

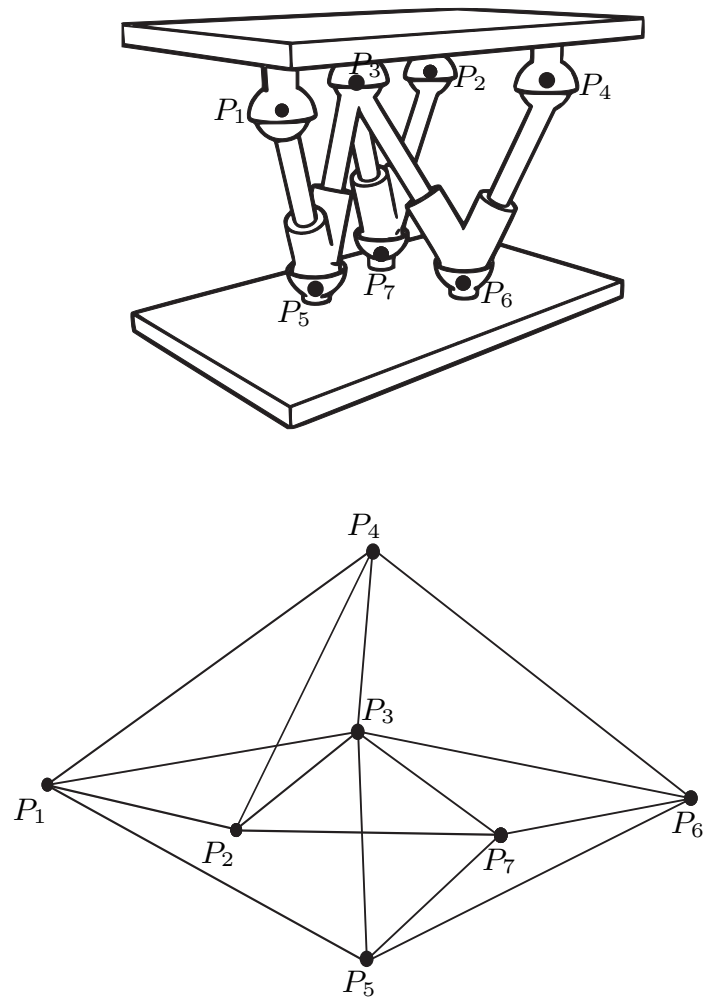

Fig. 6. The decoupled Stewart platform (top) and its equivalent bar-andjoint assembly (bottom)

$P_{j}$ and $P_{m}$, and $P_{l}$ and $P_{n}$, have been removed and then joints $P_{k}$ and $P_{p}$, and $P_{j}$ and $P_{o}$, have been merged. The closure condition of this double banana is a scalar radical equation in two variables: $s_{2,5}$ and $s_{4,7}$. After clearing radicals, an eighth-degree polynomial equation, say $\Gamma_{1}\left(s_{2,5}, s_{4,7}\right)=0$, is obtained.

2) The one resulting from removing joint $P_{6}$ : a double banana in which, as labelled in Fig. 1, bars linking $P_{i}$ and $P_{m}$, and $P_{j}$ and $P_{l}$, have been removed and then joints $P_{k}$ and $P_{p}$, and $P_{j}$ and $P_{o}$, have been merged. The application of the closure condition of this double banana is a scalar radical equation in two variables: $s_{2,5}$ and $s_{4,7}$. After clearing radicals, an eighth-degree polynomial equation, say $\Gamma_{2}\left(s_{2,5}, s_{4,7}\right)=0$, is obtained.

3) The one resulting from removing the bar joining $P_{2}$ and $P_{7}$ : a double banana in which, as labelled in Fig.1, bars linking $P_{j}$ and $P_{m}$, and $P_{l}$ and $P_{o}$, have been removed and then joints $P_{k}$ and $P_{p}$ have been merged. The closure condition of this double banana is a scalar radical equation in two variables: $s_{2,5}$ and $s_{4,7}$. After clearing radicals, a fourth-degree polynomial equation, say $\Gamma_{3}\left(s_{2,5}, s_{4,7}\right)=0$, is obtained.

By eliminating either $s_{2,5}$ or $s_{4,7}$ in the three derived polynomial equations, a final univariate polynomial can be obtained. For instance, by applying the Sylvester resultant to $\Gamma_{1}(\cdot)=0$ and $\Gamma_{3}(\cdot)=0$ to eliminate $s_{4,7}$, a 32th-degree polynomial equation in $s_{2,5}$ is obtained. This polynomial factorizes in two 16th-degree polynomials, say $\Phi\left(s_{2,5}\right)$ and $A\left(s_{2,5}\right)$. Alternatively, by applying the Sylvester resultant to $\Gamma_{2}(\cdot)=0$ and $\Gamma_{3}(\cdot)=0$ a similar result is obtained, in this case the polynomial in $s_{2,5}$ factorizes as $\Phi\left(s_{2,5}\right) B\left(s_{2,5}\right)$. Therefore, $\Phi\left(s_{2,5}\right)$, the greatest common divisor of both resulting univariate polynomials, corresponds to the univariate polynomial for the forward kinematics of the decoupled Stewart platform.

For each real root of $\Phi\left(s_{2,5}\right)$, the locations of the points in the moving platform can be determined by computing the following sequence of trilaterations:

- $\mathbf{p}_{5,3}$ from $\mathbf{p}_{5,6}$ and $\mathbf{p}_{5,7}$, then

- $\mathbf{p}_{5,2}$ from $\mathbf{p}_{5,3}$ and $\mathbf{p}_{5,7}$, then

- $\mathbf{p}_{5,1}$ from $\mathbf{p}_{5,2}$ and $\mathbf{p}_{5,3}$, and finally

- $\mathbf{p}_{1,4}$ from $\mathbf{p}_{1,2}$ and $\mathbf{p}_{1,3}$.

This procedure leads to up to eight locations for $P_{4}$. Those satisfying the distance imposed by the leg connecting $P_{4}$ and $P_{6}$ correspond to valid configurations.

\section{NUMERICAL EXAMPLE}

Let us consider the decoupled Stewart platform in Fig. 6(top). As an example, let us set $s_{1,2}=85, s_{1,3}=91$, $s_{1,4}=197, s_{2,3}=38, s_{2,4}=74, s_{3,4}=34, s_{5,6}=49$, $s_{5,7}=41$, and $s_{6,7}=34$, and squared input joints $s_{1,5}=$ $126, s_{2,7}=108, s_{3,5}=69, s_{3,6}=62, s_{3,7}=58$, and $s_{4,6}=108$. Then, proceeding as explained in the previous section, we obtain the univariate polynomial

$$
\begin{aligned}
& s_{2,5}^{16}-1665.2437 s_{2,5}^{15}+1.272210^{6} s_{2,5}^{14}-5.895210^{8} s_{2,5}^{13} \\
& +1.848710^{11} s_{2,5}^{12}-4.152510^{13} s_{2,5}^{11}+6.914610^{15} s_{2,5}^{10} \\
& -8.738410^{17} s_{2,5}^{9}+8.533810^{19} s_{2,5}^{8}-6.553310^{21} s_{2,5}^{7} \\
& +4.071510^{23} s_{2,5}^{6}-2.184810^{25} s_{2,5}^{5}+1.116510^{27} s_{2,5}^{4} \\
& -5.425610^{28} s_{2,5}^{3}+2.092310^{30} s_{2,5}^{2}-5.006610^{31} s_{2,5} \\
& +5.247910^{32} .
\end{aligned}
$$

This polynomial has 8 real roots, namely, 41.8812, 45.8373, 90.1583, 99.5174, 129.3323, 153.0000, 162.4025, and 178.4359. The corresponding solutions of the forward kinematics, for the case in which $\mathbf{p}_{5}=(2,0,0)^{T}$, $\mathbf{p}_{6}=(9,0,0)^{T}$, and $\mathbf{p}_{7}=(6,5,0)^{T}$, and assuming that $\left|\mathbf{p}_{1,3}, \mathbf{p}_{1,2}, \mathbf{p}_{1,4}\right|>0$, appear in Fig. 7 The solutions for which $\left|\mathbf{p}_{1,3}, \mathbf{p}_{1,2}, \mathbf{p}_{1,4}\right|<0$ are mirror symmetric with respect to the base to those represented in Fig. 7

\section{CONCLUSION}

Most position analysis techniques in robotics rely on the computation of a set of independent loop equations. The analysis of kinematically equivalent bar-and-joint frameworks opens a new scenario in which the elementary building blocks are the closure conditions of double bananas. Since the number of these closure conditions are typically lower than the number of scalar equations derived from sets of independent loop equations, an important simplification is gained. This has been exemplified through several examples. The presented ideas have been delivered in a rather informal way. They certainly remain to be formalized in an algorithmic setting. 


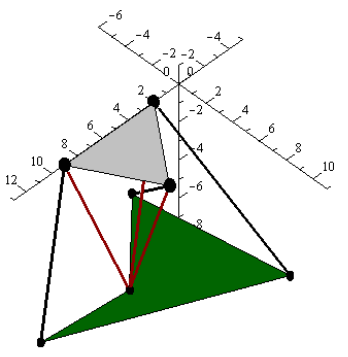

$s_{2,5}=41.8812$

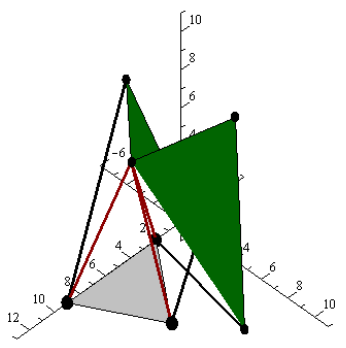

$s_{2,5}=129.3323$

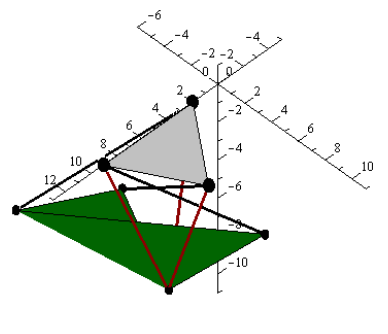

$s_{2,5}=45.8373$

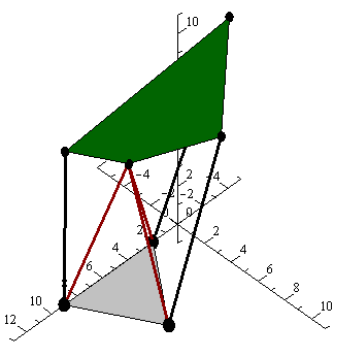

$s_{2,5}=153.0000$

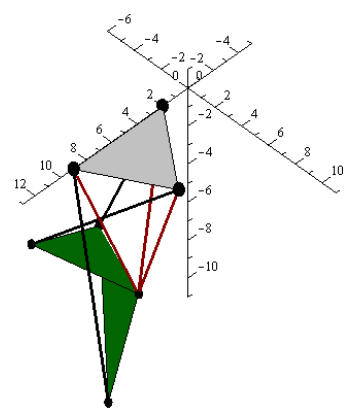

$s_{2,5}=90.1583$
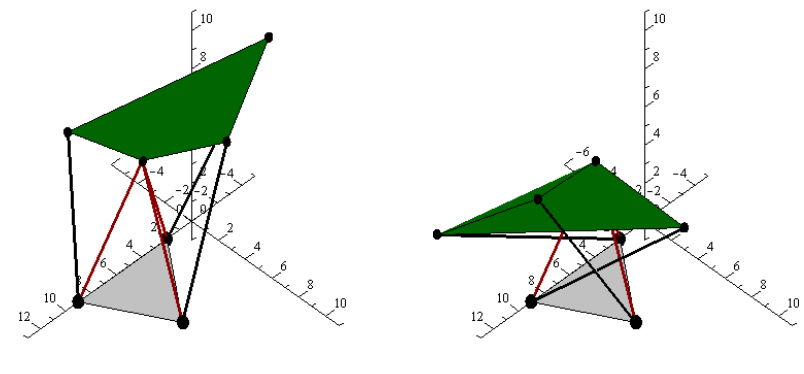

$s_{2,5}=162.4025$

Fig. 7. The forward kinematics solutions of the analyzed decoupled Stewart platform.

\section{REFERENCES}

[1] B. Servatius and H. Servatius, "Generic and abstract rigidity," in Rigidity Theory and Applications, ser. Fundamental Materials Research, M. F. Thorpe, P. M. Duxbury, and M. F. Thorpe, Eds. Springer, 2002, pp. 1-19.

[2] J. Nielsen and B. Roth, "On the kinematic analysis of robotic mechanisms," The International Journal of Robotics Research, vol. 18, no. 12 , pp. $1147-1160,1999$.

[3] J. McCarthy, 21st Century Kinematics. The 2012 NSF Workshop. Springer, 2013.

[4] K. Menger, "New foundation of Euclidean geometry," American Journal of Mathematics, vol. 53, no. 4, pp. 721-745, 1931.

[5] T. Havel, "Some examples of the use of distances as coordinates for Euclidean geometry," Journal of Symbolic Computation, vol. 11, no. 5-6, pp. $579-593,1991$.

[6] D. Michelucci and S. Foufou, "Using Cayley-Menger determinants for geometric constraint solving," in SM '04: Proceedings of the 9th ACM Symposium on Solid Modeling and Applications. Aire-la-Ville, Switzerland: Eurographics Association, 2004, pp. 285 - 290.

[7] F. Thomas and L. Ros, "Revisiting trilateration for robot localization," IEEE Transactions on Robotics, vol. 21, no. 1, pp. 93-101, 2005.

[8] J. Porta, L. Ros, and F. Thomas, "Inverse kinematics by distance matrix completion," in Proceedings of the 12th International Workshop on Computational Kinematics, 2005.

[9] P. W. M. Baili and D. Chablat, "Kinematic analysis of a family of 3R manipulators," IFToMM, Problems of Mechanics, vol. 15, pp. 27-32, 2004.

[10] D. Smith and H. Lipkin, "Higher order singularities of regional manipulators," in Proceedings of the 1993 IEEE International Conference on Robotics and Automation, 1993, pp. $194-199$ vol.1.

[11] D. Pieper and B. Roth, "The kinematics of manipulators under computer control," in Proceedings of the second international congress on theory of machines and mechanisms, 1969, pp. $159-169$.

[12] D. Smith and H. Lipkin, "Analysis of fourth order manipulator kinematics using conic sections," in Proceedings of the 1990 IEEE International Conference on Robotics and Automation, 1990, pp. 274 -278 vol.1.
[13] J. Porta, L. Ros, and F. Thomas, "On the trilaterable six-degree-offreedom parallel and serial manipulators," in Proceedings of the 2005 IEEE International Conference on Robotics and Automation, 2005, pp. 960 - 967.

[14] J. Faugère and D. Lazard, "Combinatorial classes of parallel manipulators," Mechanism and Machine Theory, vol. 30, no. 6, pp. $765-$ $776,1995$.

[15] P. Nanua, K. Waldron, and V. Murthy, "Direct kinematic solution of a Stewart platform," IEEE Transactions on Robotics and Automation, vol. 6, no. 4, pp. $438-444,1990$.

[16] M. Griffis and J. Duffy, "A forward displacement analysis of a class of Stewart platforms," Journal of Robotic Systems, vol. 6, pp. 703-720, 1989.

[17] C. Innocenti and V. Parenti-Castelli, "Direct position analysis of the Stewart platform mechanism," Mechanism and Machine Theory, vol. 25, no. 6, pp. $611-621,1990$.

[18] I. Akçali and M. Mutlu, "A novel approach in the direct kinematics of Stewart platform mechanisms with planar platforms," ASME Journal of Mechanical Dessign, vol. 128, no. 1, pp. 252-263, 2006.

[19] G. Nawratil, "Review and recent results on Stewart Gough platforms with self-motions," Applied Mechanics and Materials, vol. 162, pp. $151-160,2012$.

[20] J. Merlet, Parallel Robots, ser. Solid Mechanics and Its Applications. Kluwer Academic Publishers, 2000.

[21] R. D. Gregorio, "Kinematics of the 3-UPU wrist," Mechanism and Machine Theory, vol. 38, no. 3, pp. 253 - 263, 2003.

[22] C. Innocenti and V. Parenti-Castelli, "Direct kinematics of the 6-4 fully parallel manipulator with position and orientation uncoupled," in Robotic Systems, S. Tzafestas, Ed. Springer, 1992, vol. 10, pp. 3-10.

[23] K. Wohlhart, "Displacement analysis of the general spherical stewart platform," Mechanism and Machine Theory, vol. 29, no. 4, pp. 581 589, 1994.

[24] M. Husain and K. J. Waldron, "Direct position kinematics of the 3-11-1 Stewart platforms," Journal of Mechanical Design, vol. 116, no. 4, pp. 1102-1107, 1994. 